ALEA, Lat. Am. J. Probab. Math. Stat. 14, 361-380 (2017)

DOI: 10.30757/ALEA.v14-20

\title{
Poincaré Inequality for Dirichlet Distributions and Infinite-Dimensional Generalizations
}

\section{Shui Feng, Laurent Miclo and Feng-Yu Wang}

Department of Mathematics and Statistics, McMaster University, Hamilton, L8S 4K1, Canada.

E-mail address: shuifeng@univmail.cis.mcmaster.ca

IMT, UMR 5219, CNRS et Université Paul Sabatier, 31062 Toulouse cedex 4, France.

E-mail address: laurent.miclo@math.univ-toulouse.fr

Center for Applied Mathematics, Tianjin University, Tianjin 300072, China.

and

Department of Mathematics, Swansea University,

Singleton Park, SA2 8PP, UK.

E-mail address: wangfy@tju.edu.cn, F.Y.Wang@swansea.ac.uk

Abstract. For any $N \geq 2$ and $\alpha=\left(\alpha_{1}, \cdots, \alpha_{N+1}\right) \in(0, \infty)^{N+1}$, let $\mu_{\alpha}^{(N)}$ be the corresponding Dirichlet distribution on $\Delta^{(N)}:=\left\{x=\left(x_{i}\right)_{1 \leq i \leq N} \in[0,1]^{N}:|x|_{1}:=\right.$ $\left.\sum_{1 \leq i \leq N} x_{i} \leq 1\right\}$. We prove the Poincaré inequality

$$
\mu_{\alpha}^{(N)}\left(f^{2}\right) \leq \frac{1}{\alpha_{N+1}} \int_{\Delta^{(N)}}\left\{\left(1-|x|_{1}\right) \sum_{n=1}^{N} x_{n}\left(\partial_{n} f\right)^{2}\right\} \mu_{\alpha}^{(N)}(\mathrm{d} x)+\mu_{\alpha}^{(N)}(f)^{2},
$$

for $f \in C^{1}\left(\Delta^{(N)}\right)$, and show that the constant $\frac{1}{\alpha_{N+1}}$ is sharp. Consequently, the associated diffusion process on $\Delta^{(N)}$ converges to $\mu_{\alpha}^{(N)}$ in $L^{2}\left(\mu_{\alpha}^{(N)}\right)$ at the exponentially rate $\alpha_{N+1}$. The whole spectrum of the generator is also characterized. Moreover, the sharp Poincaré inequality is extended to the infinite-dimensional setting, and the spectral gap of the corresponding discrete model is derived.

Received by the editors March 28th, 2016; accepted April 19th, 2017.

2010 Mathematics Subject Classification. 60J60, 60H10.

Key words and phrases. Dirichlet distribution, Poincaré inequality, diffusion process, spectral gap.

Research supported by NNSFC(11431014,11626245,11626250), NSERC, and ANR-STAB-12BS01-0019. 


\section{Introduction}

Let $\mathbb{N}$ denote the set of natural numbers. For $N \in \mathbb{N}$ and any $\alpha=\left(\alpha_{1}, \cdots, \alpha_{N+1}\right)$ $\in(0, \infty)^{N+1}$, the Dirichlet distribution $\mu_{\alpha}^{(N)}$ with parameter $\alpha$ is a probability measure on the set

$$
\Delta^{(N)}:=\left\{x=\left(x_{i}\right)_{1 \leq i \leq N} \in[0,1]^{N}:|x|_{1} \leq 1\right\}
$$

with the density function

$$
\rho\left(x_{1}, \cdots, x_{N}\right):=\frac{\Gamma\left(|\alpha|_{1}\right)}{\prod_{1 \leq i \leq N+1} \Gamma\left(\alpha_{i}\right)}\left(1-|x|_{1}\right)^{\alpha_{N+1}-1} \prod_{1 \leq i \leq N} x_{i}^{\alpha_{i}-1}, \quad x \in \Delta^{(N)},
$$

where $|x|_{1}:=\sum_{1 \leq i \leq N}\left|x_{i}\right|$ for $x \in \mathbb{R}^{N}$. Obviously, $\mu_{\alpha}^{(N)}$ corresponds to the distribution

on the space

$$
\tilde{\mu}_{\alpha}^{(N+1)}(\mathrm{d} x, \mathrm{~d} y):=\mu_{\alpha}^{(N)}(\mathrm{d} x) \delta_{1-|x|_{1}}(\mathrm{~d} y)
$$

$$
\nabla^{(N+1)}:=\left\{(x, y) \in[0,1]^{N+1}: y+|x|_{1}=1\right\} .
$$

The Dirichlet distribution and its infinite-dimensional generalization arise naturally in Bayesian inference as conjugate priors for categorical distribution and infinite non-parametric discrete distributions respectively. They also arise in population genetics describing the distribution of allelic frequencies (see for instance Connor and Mosimann, 1969; Johnson, 1960; Mosimann, 1962). In particular, for a population with $N+1$ allelic types, $x_{i}(1 \leq i \leq N+1)$ stands for the relative frequency of the $i$-th allele among $N+1$ ones.

The Dirichlet distribution possesses many nice properties. We will use the following partition (or aggregation) property of $\tilde{\mu}_{\alpha}^{(N+1)}$ for $\alpha \in(0, \infty)^{N+1}$. Let $\left(X_{1}, \ldots, X_{N+1}\right)$ have law $\tilde{\mu}_{\alpha}^{(N+1)}$, let $A_{1}, A_{2}, \ldots, A_{k+1}$ be a partition of the set $\{1,2, \ldots, N+1\}$, and set

$$
Y_{j}=\sum_{r \in A_{j}} X_{r}, \beta_{j}=\sum_{r \in A_{j}} \alpha_{r}, \quad j=1, \ldots, k+1 .
$$

Then $\left(Y_{1}, \ldots, Y_{k+1}\right)$ has law $\tilde{\mu}_{\beta}^{(k+1)}$ with parameters $\beta:=\left(\beta_{1}, \ldots, \beta_{k+1}\right) \in(0, \infty)^{k+1}$. We would also like to recall the neutral property of the Dirichlet distribution. For $\left(X_{1}, \cdots, X_{N}\right)$ having law $\mu_{\alpha}^{(N)}$, we define

$$
U_{1}=X_{1}, U_{i}=\frac{X_{i}}{1-X_{1}-\ldots-X_{i-1}}, \quad 2 \leq i \leq N .
$$

Then $U_{i}$ is a beta random variable with parameters $\left(\alpha_{i}, \alpha_{i+1}+\ldots+\alpha_{N+1}\right)$ and $U_{1}, \ldots, U_{N}$ are independent. This leads to the following representation of the random variable with law $\mu_{\alpha}^{(N)}$ :

$$
\left(X_{1}, X_{2}, \ldots, X_{N}\right)=\left(U_{1}, U_{2}\left(1-U_{1}\right), \ldots, U_{N} \prod_{i=1}^{N-1}\left(1-U_{i}\right)\right) .
$$

A well known construction of the Dirichlet distribution is through a Pólya urn scheme (cf. Blackwell and MacQueen, 1973). More specifically, consider an urn containing $N+1$ balls of different colors labelled by $1,2, \ldots, N+1$. The initial mass of the $i$-colored ball is $\alpha_{i}$. Balls are drawn from the urn sequentially. The chance of a particular colored ball being selected is proportional to the total mass of that colored 
balls inside the urn. After each selection, the ball is returned with an additional ball of same color and mass one. The relative weight of different colored balls inside the urn will eventually converge to a Dirichlet vector $\left(X_{1}, X_{2}, \ldots, X_{N+1}\right)$.

Several diffusion processes have been proposed and studied where the stationary distribution is the Dirichlet distribution.

Exploring the property of right neutrality, a GEM diffusion is introduced in Feng and Wang (2007) and studied further in Feng and Wang (2016). This is a reversible diffusion with Dirichlet distribution as the reversible measure. The infinite-dimensional generalization of the model is also reversible and the reversible measure is the GEM distribution (see Ewens, 2004).

The most studied diffusion model is the Wright-Fisher diffusion (see Epstein and Mazzeo, 2010; Miclo, 2003a,b; Stannat, 2000). This is a diffusion approximation to the Wright-Fisher Markov chain model in population genetics. The Markov chain models the evolution of a population of individuals of finite number of different types. The evolution is driven by mutation (deterministic component) and genetic drift or random sampling (random component). Each individual follows a deterministic path of mutation. The random sampling involves the exchange of types of any pair of individuals in the population. The involvement of every pair in the sampling process resulted in a lot of randomness in the system. The diffusion arises as the population size increases while the mutation and sampling rates are scaled appropriately. It is reversible with respect to the Dirichlet distribution. Exploring the exchangeable structure embedded in the system, one is able to obtain the Infinite-dimensional generalizations of this model including the infinitely-manyneutral-alleles model (Ethier and Kurtz, 1981) and the Fleming-Viot process with parent independent mutation (Fleming and Viot, 1979; Ethier and Kurtz, 1993).

In this paper, we focus on a diffusion process introduced in Jacobsen (2001, (2.44)) (see also Bakosi and Ristorcelli, 2013), which solves the following SDE on $\Delta^{(N)}$ :

$\mathrm{d} X_{i}(t)=\left\{\alpha_{i}(1-|X(t)|)-\alpha_{N+1} X_{i}(t)\right\} \mathrm{d} t+\sqrt{2\left(1-|X(t)|_{1}\right) X_{i}(t)} \mathrm{d} B_{i}(t), \quad 1 \leq i \leq N$,

where $B(t):=\left(B_{1}(t), \cdots, B_{N}(t)\right)$ is the $d$-dimensional Brownian motion.

The evolution in this model also contains a deterministic component and a random component. For $N=1$, the model is the same as the Wright-Fisher diffusion. For $N \geq 2$, the deterministic part is very similar to the mutation in the WrightFisher model. But the random sampling does not involve all individual pairs. Instead each sampling is between one individual and one fixed individual. This clearly reduces the randomness in the system. But it will turn out that the model possesses many features of the Wright-Fisher diffusion.

More specifically, we will show that the Markov semigroup $P_{t}^{\alpha}$ associated to (1.1) is symmetric in $L^{2}\left(\mu_{\alpha}^{(N)}\right)$; that is,

$$
\int_{\Delta^{(N)}} f L_{\alpha}^{(N)} g \mathrm{~d} \mu_{\alpha}^{(N)}=\int_{\Delta^{(N)}} g L_{\alpha}^{(N)} f \mathrm{~d} \mu_{\alpha}^{(N)}, \quad f, g \in C^{2}\left(\mathbb{R}^{N}\right)
$$

holds for

$$
L_{\alpha}^{(N)}(x):=\sum_{1 \leq n \leq N}\left(x_{n}\left(1-|x|_{1}\right) \partial_{n}^{2}+\left\{\alpha_{n}\left(1-|x|_{1}\right)-\alpha_{N+1} x_{n}\right\} \partial_{n}\right)
$$


being the generator of $P_{t}^{\alpha}$, where $\partial_{n}:=\frac{\partial}{\partial x_{n}}$. So, $\left(L_{\alpha}^{(N)}, C^{2}\left(\Delta^{(N)}\right)\right)$ is closable in $L^{2}\left(\mu_{\alpha}^{(N)}\right)$ and its closure $\left(L_{\alpha}^{(N)}, \mathcal{D}\left(L_{\alpha}\right)\right)$ is a negative definite self-adjoint operator.

Moreover, since

$$
L_{\alpha}^{(N)}(f g)(x)=\left(f L_{\alpha}^{(N)} g+g L_{\alpha}^{(N)} f\right)(x)+2\left(1-|x|_{1}\right) \sum_{n=1}^{N} x_{n}\left\{\left(\partial_{n} f\right)\left(\partial_{n} g\right)\right\}(x),
$$

(1.2) implies the integration by parts formula

$$
\begin{aligned}
-\int_{\Delta^{(N)}} f L_{\alpha}^{(N)} g \mathrm{~d} \mu_{\alpha}^{(N)} & =\int_{\Delta^{(N)}}\left\{\left(1-|x|_{1}\right) \sum_{n=1}^{N} x_{n}\left\{\left(\partial_{n} f\right)\left(\partial_{n} g\right)\right\}(x)\right\} \mu_{\alpha}^{(N)}(\mathrm{d} x) \\
& =: \mathcal{E}_{\alpha}^{(N)}(f, g), \quad f, g \in C^{2}\left(\Delta^{(N)}\right) .
\end{aligned}
$$

Therefore, $\left(\mathcal{E}_{\alpha}^{(N)}, C^{2}\left(\Delta^{(N)}\right)\right)$ is closable in $L^{2}\left(\mu_{\alpha}^{(N)}\right)$ whose closure $\left(\mathcal{E}_{\alpha}^{(N)}, \mathcal{D}\left(\mathcal{E}_{\alpha}^{(N)}\right)\right)$ is a symmetric Dirichlet form on $L^{2}\left(\mu_{\alpha}^{(N)}\right)$, and it is easy to see that this Dirichlet form is associated to the Markov semigroup $P_{t}^{\alpha}$.

Finally, the spectral gap of $L_{\alpha}^{(N)}$ is characterized as

$$
\operatorname{gap}\left(L_{\alpha}^{(N)}\right)=\inf \left\{\mathcal{E}_{\alpha}^{(N)}(f, f): f \in \mathcal{D}\left(\mathcal{E}_{\alpha}^{(N)}\right), \mu_{\alpha}^{(N)}(f)=0, \mu_{\alpha}^{(N)}\left(f^{2}\right)=1\right\} .
$$

It is known that when $N=1$ we have $\operatorname{gap}\left(L_{\alpha}^{(N)}\right)=\alpha_{1}+\alpha_{2}$, see e.g. Stannat (2000). So, in the following we only consider $N \geq 2$.

Let $\mathcal{K}$ be the set of elements of the form $k:=\left(k_{1}, k_{2}, \ldots, k_{r}, k_{r+1}\right) \in \mathbb{Z}_{+}^{r+1}$, where $r \in \mathbb{Z}_{+}$and $0 \leq k_{1}<k_{2}<\cdots<k_{r}<k_{r+1}$. Define mappings $K, D: \mathcal{K} \rightarrow[0, \infty)$ as follows: $\forall k:=\left(k_{1}, k_{2}, \ldots, k_{r}, k_{r+1}\right) \in \mathcal{K}$,

$$
\begin{aligned}
& K(k):=2\left(k_{1}+\cdots k_{r}\right)+r \tilde{\alpha}+k_{r+1} \alpha_{N+1}, \\
& D(k):=\sum_{1 \leq l \leq r} C\left(N, k_{l}\right)+\sum_{1 \leq l \leq k_{r+1}-1, l \notin\left\{k_{1}, k_{2}, \ldots, k_{r}\right\}}\{C(N, l+1)-C(N, l)\} .
\end{aligned}
$$

Then our first result provides a complete characterization of the spectrum $\Lambda$ for $-L_{\alpha}^{(N)}$, in particular, the spectral gap is given.

Theorem 1.1. Let $N \geq 2$. Then $P_{t}^{\alpha}$ is symmetric in $L^{2}\left(\mu_{\alpha}^{(N)}\right)$ and the spectrum of $-L_{\alpha}^{(N)}$ is

$$
\Lambda=\{K(k)[D(k)]: k \in \mathcal{K}\},
$$

where $\lambda[m]$ means that $\lambda$ is an eigenvalue having multiplicity $m$. Consequently, $\operatorname{gap}\left(L_{\alpha}^{(N)}\right)=\alpha_{N+1}$, so that $P_{t}^{\alpha}$ converge to $\mu_{\alpha}^{(N)}$ exponentially fast in $L^{2}\left(\mu_{\alpha}^{(N)}\right)$ :

$$
\left\|P_{t}^{\alpha}-\mu_{\alpha}^{(N)}\right\|_{L^{2}\left(\mu_{\alpha}^{(N)}\right)} \leq \mathrm{e}^{-\alpha_{N+1} t}, \quad t \geq 0
$$

and the sharp Poincaré inequality for $\left(\mathcal{E}_{\alpha}^{(N)}, \mathcal{D}\left(\mathcal{E}_{\alpha}^{(N)}\right)\right)$ is

$$
\mu_{\alpha}^{(N)}\left(f^{2}\right) \leq \frac{1}{\alpha_{N+1}} \mathcal{E}_{\alpha}^{(N)}(f, f), \quad f \in \mathcal{D}\left(\mathcal{E}_{\alpha}^{(N)}\right), \mu_{\alpha}^{(N)}(f)=0 .
$$


Remark 1.1. (1) One may wonder if there holds stronger functional inequalities or not, for instance, the log-Sobolev inequality, or more generally the super Poincaré inequality introduced in Wang (2000a). When $N=1$, it is easy to see that when $\alpha_{1}, \alpha_{2} \geq \frac{1}{4}$ and $\alpha_{1}+\alpha_{2}>\frac{1}{2}$, the Bakry and Émery (1984) criterion holds so that the log-Sobolev inequality is valid. Indeed, Stannat (2000, Lemma 2.7) has proved the following log-Sobolev inequality for $N=1$ and any $\alpha_{1}, \alpha_{2}>0$ :

$$
\mu_{\alpha}^{(1)}\left(f^{2} \log f^{2}\right) \leq \frac{320}{\alpha_{1} \wedge \alpha_{2}} \int_{0}^{1} x(1-x) f^{\prime}(x)^{2} \mu_{\alpha}^{(1)}(\mathrm{d} x), \quad f \in C_{b}^{1}(0,1) .
$$

This result was extended in Feng and Wang (2007) to the infinite-dimensional GEM distribution on

$$
\Delta_{\infty}:=\left\{x \in[0,1]^{\mathbb{N}}: \sum_{i=1}^{\infty} x_{i}=1\right\} .
$$

However, for the present model the log-Sobolev inequality is unknown for $N \geq 2$. On the other hand, since the spectrum of $L_{\alpha}^{(N)}$ is discrete due to Theorem 1.1, by Wang (2000b, Theorem 3.1), the super Poincaré inequality

$$
\mu_{\alpha}^{(N)}\left(f^{2}\right) \leq r \mathcal{E}_{\alpha}^{(N)}(f, f)+\beta(r) \mu_{\alpha}^{(N)}(|f|)^{2}, \quad r>0, f \in \mathcal{D}\left(\mathcal{E}_{\alpha}^{(N)}\right)
$$

holds for some $\beta:(0, \infty) \rightarrow(0, \infty)$. But in the moment we do not have any estimate on the rate function $\beta$. Note that the log-Sobolev inequality holds if and only if $\beta(r)$ in the super Poincaré inequality satisfies $\beta(r) \leq \mathrm{e}^{-c r^{-1}}$ for some constant $c>0$ and small $r>0$, see Wang (2000a, Corollary 3.3).

(2) The operator $L_{\alpha}^{(N)}$ we considered is a special case of the following general operator on $\mathbb{R}_{+}^{n} \times \mathbb{R}^{m}$ investigated in Epstein and Mazzeo (2013):

$$
L=\sum_{i=1}^{n} a_{i i} x_{i} \partial_{x_{i}}^{2}+\sum_{1 \leq i \neq j \leq n} x_{i} x_{j} a_{i j} \partial_{x_{i} x_{j}}^{2}+\sum_{i=1}^{n} \sum_{j=1}^{m} x_{i} b_{i k} \partial_{x_{i} y_{k}}^{2}+\sum_{k, l=1}^{m} c_{k l} \partial_{y_{k} y_{l}}^{2}+V
$$

where $\left(a_{i j}\right)$ and $\left(c_{k l}\right)$ are symmetric matrices, $V$ is a vector field. Integral type Hölder/derivative estimates in both time and space variables are presented in Epstein and Mazzeo (2013) for the heat kernel.

Next, we extend Theorem 1.1 to the infinite-dimensional setting. Consider the infinite-dimensional simplex

$$
\Delta^{(\infty)}:=\left\{x \in[0,1]^{\mathbb{N}}:|x|_{1}=\sum_{i=1}^{\infty} x_{i} \leq 1\right\}
$$

which is equipped with the $L^{1}$-metric $|x-y|_{1}$. Let $\alpha \in(0, \infty)^{\mathbb{N}}$ with $|\alpha|_{1}=$ $\sum_{i=1}^{\infty} \alpha_{i}<\infty$, and let $\alpha_{\infty}>0$ which refers to $\alpha_{N+1}$ in the finite-dimensional case as $N \rightarrow \infty$. Let

$$
\alpha^{(n)}=\left(\alpha_{1}, \cdots, \alpha_{n-1}, \sum_{i \geq n} \alpha_{i}, \alpha_{\infty}\right) \in(0, \infty)^{n+1}, \quad n \geq 1 .
$$

Then for any $n \geq 1$,

$$
\mu_{\alpha, \alpha_{\infty}}^{(n)}(\mathrm{d} x):=\mu_{\alpha^{(n)}}^{(n)}\left(\mathrm{d} x_{1}, \cdots, \mathrm{d} x_{n}\right) \prod_{i=n+1}^{\infty} \delta_{0}\left(\mathrm{~d} x_{i}\right)
$$


is a probability measure on $\Delta^{(\infty)}$. We will prove that when $n \rightarrow \infty$ these measures converges weakly to a probability measure $\mu_{\alpha, \alpha_{\infty}}^{(\infty)}$ on $\Delta^{(\infty)}$, which is the infinitedimensional generalization of Dirichlet distribution with parameters $\left(\alpha, \alpha_{\infty}\right)$.

The following result extends Theorem 1.1 to the infinite-dimensional setting, for which we introduce the class of $C^{p}$-cylindrical functions for $p \geq 1$ :

$$
\mathcal{F} C^{p}:=\left\{\Delta^{(\infty)} \ni x:=\left(x_{i}\right)_{i \geq 1} \mapsto f\left(x_{1}, \cdots, x_{n}\right): n \geq 1, f \in C^{p}\left(\mathbb{R}^{n}\right)\right\} .
$$

Theorem 1.2. Let $\alpha \in(0, \infty)^{\mathbb{N}}$ with $|\alpha|_{1}<\infty$ and let $\alpha_{\infty}>0$.

(1) The sequence $\left\{\mu_{\alpha, \alpha_{\infty}}^{(n)}\right\}_{n \geq 1}$ converges weakly to a probability measure $\mu_{\alpha, \alpha_{\infty}}^{(\infty)}$ on $\Delta(\infty)$

(2) The form

$$
\mathcal{E}_{\alpha, \alpha_{\infty}}^{(\infty)}(f, g):=\int_{\Delta(\infty)}\left\{\left(1-|x|_{1}\right) \sum_{n=1}^{\infty} x_{n}\left(\partial_{n} f\right) \partial_{n} g\right\}(x) \mu_{\alpha, \alpha_{\infty}}^{(\infty)}(\mathrm{d} x), \quad f, g \in \mathcal{F} C^{1}
$$

is closable in $L^{2}\left(\mu_{\alpha, \alpha_{\infty}}^{(\infty)}\right)$ whose closure is a symmetric Dirichlet form. The generator $\left(L_{\alpha, \alpha_{\infty}}^{(\infty)}, \mathcal{D}\left(L_{\alpha, \alpha_{\infty}}^{(\infty)}\right)\right)$ of the Dirichlet form satisfies $\mathcal{F} C^{2} \subset \mathcal{D}\left(L_{\alpha, \alpha_{\infty}}^{(\infty)}\right)$ and

$L_{\alpha, \alpha_{\infty}}^{(\infty)} f(x)=\sum_{n=1}^{\infty}\left(x_{n}\left(1-|x|_{1}\right) \partial_{n}^{2} f(x)+\left\{\alpha_{n}\left(1-|x|_{1}\right)-\alpha_{\infty} x_{n}\right\} \partial_{n} f(x)\right), f \in \mathcal{F} C^{2}$.

(3) The generator $L_{\alpha, \alpha_{\infty}}^{(\infty)}$ has spectral gap $\operatorname{gap}\left(L_{\alpha, \alpha_{\infty}}^{(\infty)}\right)=\alpha_{\infty}$. Consequently, the associated Markov semigroup $P_{t}^{\alpha, \alpha_{\infty}}$ converges to $\mu_{\alpha, \alpha_{\infty}}^{(\infty)}$ exponentially fast in $L^{2}\left(\mu_{\alpha, \alpha_{\infty}}^{(\infty)}\right)$ :

$$
\left\|P_{t}^{\alpha, \alpha_{\infty}}-\mu_{\alpha, \alpha_{\infty}}^{(\infty)}\right\|_{L^{2}\left(\mu_{\alpha, \alpha}^{(\infty)}\right)} \leq \mathrm{e}^{-\alpha_{\infty} t}, \quad t \geq 0
$$

and the sharp Poincaré inequality is

$$
\mu_{\alpha, \alpha_{\infty}}^{(\infty)}\left(f^{2}\right) \leq \frac{1}{\alpha_{\infty}} \mathcal{E}_{\alpha, \alpha_{\infty}}^{(\infty)}(f, f), \quad f \in \mathcal{F} C^{1}, \mu_{\alpha, \alpha_{\infty}}^{(\infty)}(f)=0
$$

Finally, the next result shows that the diffusion process generated by $L_{\alpha, \alpha_{\infty}}^{(\infty)}$ is the weak limit of the $L_{\alpha, \alpha_{\infty}}^{(n)}$-diffusion process as $n \rightarrow \infty$, where

$$
L_{\alpha, \alpha_{\infty}}^{(n)}:=\sum_{i=1}^{n}\left\{\left[\alpha_{i}\left(1-\sum_{i=1}^{n} x_{i}\right)-\alpha_{\infty} x_{i}\right] \partial_{i}+2\left(1-\sum_{i=1}^{n} x_{i}\right) x_{i} \partial_{i}^{2}\right\} .
$$

For any $x \in \Delta^{(\infty)}$ and $T>0$, let $P_{x, T}^{(n)}$ be the distribution of the diffusion process generated by $L_{\alpha, \alpha_{\infty}}^{(n)}$ with initial point $x^{(n)}:=\left(x_{1}, \cdots, x_{n-1}, \sum_{j \geq n} x_{j}\right)$. Embedding $\Delta^{(n)}$ into $\Delta^{(\infty)}$ by setting $z_{i}=0$ for $z \in \Delta^{(n)}$ and $i \geq n+1$, we regard $P_{x, T}^{(n)}$ as a probability measure on $\Omega_{T}:=C\left([0, T] ; \Delta_{\infty}\right)$ equipped with the uniform norm $\|\xi\|_{1, \infty}:=\sup _{t \in[0, T]}|\xi(t)|_{1}$.

Theorem 1.3. For any $x \in \Delta^{(\infty)}$ and $T>0, P_{x, T}^{(n)}$ converges weakly to a probability measure $P_{x, T}^{(\infty)}$ on $\Omega_{T}$. Moreover, $P_{x, T}^{(\infty)}$ solves the martingale problem of $L_{\alpha, \alpha_{\infty}}^{(\infty)}$ : for any $f \in \mathcal{F} C^{2}$, the coordinate process $X(t)(\omega):=\omega(t)$ and the natural filtration $\mathcal{F}_{t}:=\sigma\left(\omega_{s}: s \in[0, t]\right)$

$$
f(X(t))-\int_{0}^{t} L_{\alpha, \alpha_{\infty}}^{(\infty)} f(X(s)) \mathrm{d} s, \quad t \in[0, T]
$$


is a martingale under $P_{x, T}^{(\infty)}$.

The spectral gap of $L_{\alpha}^{(N)}$ is obtained in Section 2 by a straightforward calculation linking the gap to the eigenvalue of a matrix. Exploring this link further, we are able to identify the whole spectrum of $L_{\alpha}^{(N)}$ and to prove Theorem 1.1 in Section 3 . Even though the spectral gap of $L_{\alpha}^{(N)}$ can be obtained as part of the whole spectrum, we present the separate proof in Section 2 due to its own interests. The proofs of Theorems 1.2 and 1.3 are presented in Section 4. Finally, to better understand the biological context of the study, we introduce in Section 5 a discrete model involving immigration, emigration and sampling, which approximates the diffusion process solving (1.1).

\section{The spectral gap of $L_{\alpha}^{(N)}$}

We first prove (1.2) which implies the symmetry of $P_{t}^{\alpha}$ in $L^{2}\left(\mu_{\alpha}^{(N)}\right)$. Since smooth functions on $\Delta^{(N)}$ are uniformly approximated by polynomials up to second order derivatives, it suffices to consider $f, g \in \mathcal{P}_{\infty}$, the set of all polynomials on $\Delta^{(N)}$. Let

$$
A_{\alpha}^{(n)}=x_{n}\left(1-|x|_{1}\right) \partial_{n}^{2}+\left\{\alpha_{n}\left(1-|x|_{1}\right)-\alpha_{N+1} x_{n}\right\} \partial_{n}, \quad 1 \leq n \leq N
$$

Then (1.2) follows from

$$
\begin{aligned}
& \int_{\Delta^{(N)}}\left(\prod_{1 \leq i \leq N} x_{i}^{p_{i}}\right) A_{\alpha}^{(n)}\left(\prod_{1 \leq i \leq N} x_{i}^{q_{i}}\right) \mu_{\alpha}^{(N)}(\mathrm{d} x) \\
& =\int_{\Delta^{(N)}}\left(\prod_{1 \leq i \leq N} x_{i}^{q_{i}}\right) A_{\alpha}^{(n)}\left(\prod_{1 \leq i \leq N} x_{i}^{p_{i}}\right) \mu_{\alpha}^{(N)}(\mathrm{d} x)
\end{aligned}
$$

for $p_{i}, q_{i} \in \mathbb{Z}_{+}, 1 \leq i \leq N$. Letting $p_{N+1}=q_{N+1}=0$ and $C=\frac{\Gamma\left(|\alpha|_{1}\right)}{\prod_{1 \leq i \leq N+1} \Gamma\left(\alpha_{i}\right)}$, and simply denote $x_{N+1}=1-|x|_{1}$, we have

$$
\begin{aligned}
& \int_{\Delta^{(N)}}\left(\prod_{1 \leq i \leq N} x_{i}^{p_{i}}\right) A_{\alpha}^{(n)}\left(\prod_{1 \leq i \leq N} x_{i}^{q_{i}}\right) \mu_{\alpha}^{(N)}(\mathrm{d} x) \\
& =C \int_{\Delta^{(N)}}\left(\prod_{1 \leq i \neq n \leq N+1} x_{i}^{p_{i}+q_{i}+\alpha_{i}-1}\right) x_{n}^{p_{n}+\alpha_{n}-1} A_{\alpha}^{(n)} x_{n}^{q_{n}} \mathrm{~d} x \\
& =C q_{n}\left\{\left(q_{n}+\alpha_{n}-1\right) \int_{\Delta^{(N)}}\left(\prod_{1 \leq i \neq n \leq N+1} x_{i}^{p_{i}+q_{i}+\alpha_{i}-1}\right) x_{N+1} x_{n}^{p_{n}+q_{n}+\alpha_{n}-2} \mathrm{~d} x\right. \\
& \left.\quad-\alpha_{N+1} \int_{\Delta^{(N)}}\left(\prod_{1 \leq i \leq N+1} x_{i}^{p_{i}+q_{i}+\alpha_{i}-1}\right) \mathrm{d} x\right\} \\
& =\frac{C q_{n} \prod_{1 \leq i \neq n \leq N+1} \Gamma\left(\alpha_{i}+p_{i}+q_{i}\right)}{\Gamma\left(\sum_{1 \leq i \leq N+1}\left(\alpha_{i}+p_{i}+q_{i}\right)\right)} \\
& \times\left(\left(q_{n}+\alpha_{n}-1\right) \Gamma\left(\alpha_{N+1}+1\right) \Gamma\left(p_{n}+q_{n}+\alpha_{n}-1\right)\right. \\
& \left.\quad-\alpha_{N+1} \Gamma\left(\alpha_{N+1}\right) \Gamma\left(p_{n}+q_{n}+\alpha_{n}\right)\right) \\
& =-\frac{C \Gamma\left(\alpha_{N+1}+1\right) \prod_{1 \leq i \neq n \leq N+1} \Gamma\left(\alpha_{i}+p_{i}+q_{i}\right)}{\Gamma\left(\sum_{1 \leq i \leq N+1}\left(\alpha_{i}+p_{i}+q_{i}\right)\right)} p_{n} q_{n} \Gamma\left(p_{n}+q_{n}+\alpha_{n}-1\right),
\end{aligned}
$$


where the last step is due to the identity $\Gamma(s+1)=s \Gamma(s), s>0$. Since the result is symmetric in $\left(p_{n}, q_{n}\right)$, it implies (2.1).

For any $d \in \mathbb{N}$, let $\mathcal{P}_{d}$ be the space of all polynomials in $\mathcal{P}_{\infty}$ whose total degrees are less than or equal to $d$. Let $\mathcal{P}_{0, d}=\left\{f \in \mathcal{P}_{d}: \mu_{\alpha}^{(N)}(f)=0\right\}$. It is well known that $\mathcal{P}_{\infty}:=\cup_{d \geq 1} \mathcal{P}_{d}$ is dense in $C_{b}^{1}\left(\Delta^{(N)}\right)$, so that $\mathcal{P}_{0, \infty}:=\cup_{d \geq 1} \mathcal{P}_{0, d}$ is dense in

$$
\mathcal{D}_{0}:=\left\{f \in \mathcal{D}\left(\mathcal{E}_{\alpha}^{(N)}\right): \mu_{\alpha}^{(N)}(f)=0\right\}
$$

under the Sobolev norm $\|f\|_{1,2}:=\sqrt{\mu_{\alpha}^{(N)}\left(f^{2}\right)+\mathcal{E}_{\alpha}^{(N)}(f, f)}$.

To characterize the $\operatorname{gap}\left(L_{\alpha}^{(N)}\right)$, we decompose the spectrum of $L_{\alpha}^{(N)}$ in terms of the degree of polynomials. Obviously, every $\mathcal{P}_{0, d}$ is an invariant space of $L_{\alpha}^{(N)}$. Let $\mathcal{Q}_{1}=\mathcal{P}_{0,1}$ and

$$
\mathcal{Q}_{d}=\left\{f \in \mathcal{P}_{0, d}: \mu_{\alpha}^{(N)}(f g)=0 \text { for all } g \in \mathcal{P}_{d-1}\right\}, \quad d \geq 2 .
$$

Then, by the symmetry of $L_{\alpha}^{(N)}$ in $L^{2}\left(\mu_{\alpha}^{(N)}\right)$, every $\mathcal{Q}_{d}$ is an invariant space of $L_{\alpha}^{(N)}$ as well. Thus, letting $\pi_{d}: \mathcal{P}_{\infty} \rightarrow \mathcal{P}_{d}$ be the orthogonal projection with respect to the inner product in $L^{2}\left(\mu_{\alpha}^{(N)}\right)$, we have

$$
L_{\alpha}^{(N)} \pi_{d} f=\pi_{d} L_{\alpha}^{(N)} f, \quad d \geq 1, f \in \mathcal{P}_{\infty} .
$$

Therefore, to characterize the spectrum of $L_{\alpha}^{(N)}$ it suffices to consider that of $\left.L_{\alpha}^{(N)}\right|_{\mathcal{Q}_{i}}$, the restriction of $L_{\alpha}^{(N)}$ on $\mathcal{Q}_{i}$, for every $i \geq 1$.

Let $d \geq 2$. To characterize the spectrum of $\left.L_{\alpha}^{(N)}\right|_{\mathcal{Q}_{d}}$, let

$$
K_{d}=\left\{k=\left(k_{1}, \cdots, k_{N}\right) \in \mathbb{Z}_{+}^{N}: \sum_{1 \leq i \leq N} k_{i}=d\right\} .
$$

For any $k \in K_{d}$, let $x^{k}=\prod_{1 \leq i \leq N} x_{i}^{k_{i}}$. Then

$$
\mathcal{Q}_{d}=\left\{\sum_{k \in K_{d}} c_{k} x^{k}-\pi_{d-1} \sum_{k \in K_{d}} c_{k} x^{k}: c:=\left(c_{k}\right)_{k \in K_{d}} \in \mathbb{R}^{K_{d}}\right\} .
$$

We define the $K_{d} \times K_{d}$-matrix $M_{d}$ by letting

$$
M_{d}\left(k, k^{\prime}\right)=\left\{\begin{array}{l}
d \alpha_{N+1}+\sum_{1 \leq n \leq N}\left(k_{n}+\alpha_{n}-1\right) k_{n}, \quad \text { if } k=k^{\prime}, \\
\left(k_{n}+\alpha_{n}\right)\left(k_{n}+1\right), \quad \text { if } k^{\prime}=k+e_{n}-e_{m}, 1 \leq n \neq m \leq N, \\
0, \quad \text { otherwise, }
\end{array}\right.
$$

where $\left\{e_{n}\right\}_{1 \leq n \leq N}$ is the canonical orthonormal basis on $\mathbb{R}^{N}$. We first identify eigenvalues of $\left.L_{\alpha}^{(N)}\right|_{\mathcal{Q}_{d}}$ with those of $M_{d}$.

Lemma 2.1. For any $d \geq 2, \lambda$ is an eigenvalue of $-\left.L_{\alpha}^{(N)}\right|_{\mathcal{Q}_{d}}$ if and only if it is an eigenvalue of $M_{d}$. Consequently, $-\left.L_{\alpha}^{(N)}\right|_{\mathcal{Q}_{d}} \geq\left(d \alpha_{N+1}\right) I_{\mathcal{Q}_{d}}$, where $I_{\mathcal{Q}_{d}}$ is the identity operator on $\mathcal{Q}_{d}$.

Proof: (1) Let $\lambda$ be an eigenvalue of $-L_{\alpha}^{(N)}$ on $\mathcal{Q}_{d}$. By (2.3) and (2.2), there exists $0 \neq c \in \mathbb{R}^{K_{d}}$ such that

$$
\sum_{k \in K_{d}} c_{k}\left(L_{\alpha}^{(N)} x^{k}-\pi_{d-1} L_{\alpha}^{(N)} x^{k}\right)=-\lambda \sum_{k \in K_{d}} c_{k}\left(x^{k}-\pi_{d-1} x^{k}\right) .
$$


Obviously,

$$
\begin{aligned}
& L_{\alpha}^{(N)} x^{k}-\sum_{1 \leq n \leq N}\left(x_{n} \partial_{n}^{2}+\alpha_{n} \partial_{n}\right) x^{k} \\
& =-\left(\sum_{1 \leq n, m \leq N} x_{n} x_{m} \partial_{n}^{2} x^{k}+\sum_{1 \leq n, m \leq N} x_{m} \alpha_{n} \partial_{n} x^{k}+\alpha_{N+1} \sum_{1 \leq n \leq N} x_{n} \partial_{n} x^{k}\right) \\
& =-\left(\sum_{n, m \leq N} k_{n}\left(k_{n}-1\right) x^{k-e_{n}+e_{m}}+\sum_{1 \leq n, m \leq N} \alpha_{n} k_{n} x^{k-e_{n}+e_{m}}+\alpha_{N+1} \sum_{1 \leq n \leq N} k_{n} x^{k}\right) \\
& =-\left(\sum_{1 \leq n, m \leq N} k_{n}\left(k_{n}-1\right) x^{k-e_{n}+e_{m}}+\sum_{1 \leq n, m \leq N} \alpha_{n} k_{n} x^{k-e_{n}+e_{m}}+d \alpha_{N+1} x^{k}\right) .
\end{aligned}
$$

By the change of variables $k^{\prime}:=k-e_{n}+e_{m}$, we obtain

$$
\begin{aligned}
& \sum_{k \in K_{d}} c_{k} \sum_{1 \leq n, m \leq N} \alpha_{n} k_{n} x^{k-e_{n}+e_{m}} \\
& =\sum_{k \in K_{d}} c_{k} \sum_{1 \leq n \neq m \leq N} \alpha_{n} k_{n} x^{k-e_{n}+e_{m}}+\sum_{k \in K_{d}} c_{k} \sum_{1 \leq n \leq N} \alpha_{n} k_{n} x^{k} \\
& =\sum_{k \in K_{d}} \sum_{1 \leq n \neq m \leq N} c_{k^{\prime}+e_{n}-e_{m}} \alpha_{n}\left(k^{\prime}+e_{n}-e_{m}\right)(n) x^{k^{\prime}}+\sum_{k \in K_{d}} c_{k} \sum_{1 \leq n \leq N} \alpha_{n} k_{n} x^{k} \\
& =\sum_{k \in K_{d}} \sum_{1 \leq n \neq m \leq N} \alpha_{n}\left(k_{n}+1\right) c_{k+e_{n}-e_{m}} x^{k}+\sum_{k \in K_{d}} \sum_{1 \leq n \leq N} \alpha_{n} k_{n} c_{k} x^{k} .
\end{aligned}
$$

Similarly,

$$
\begin{aligned}
& \sum_{k \in K_{d}} c_{k} \sum_{1 \leq n, m \leq N} k_{n}\left(k_{n}-1\right) x^{k-e_{n}+e_{m}} \\
& =\sum_{k \in K_{d}} \sum_{1 \leq n \neq m \leq N} k_{n}\left(k_{n}+1\right) c_{k+e_{n}-e_{m}} x^{k}+\sum_{k \in K_{d}} c_{k} \sum_{1 \leq n \leq N} k_{n}\left(k_{n}-1\right) x^{k} .
\end{aligned}
$$

Combining these together leads to

$$
\sum_{k \in K_{d}} c_{k} L_{\alpha}^{(N)} x^{k}=\sum_{k \in K_{d}} c_{k} \sum_{1 \leq n \leq N}\left(x_{n} \partial_{n}^{2}+\delta_{n} \partial_{n}\right) x^{k}-\sum_{k, k^{\prime} \in K_{d}} M_{d}\left(k, k^{\prime}\right) c_{k^{\prime}} x^{k} .
$$

Substituting this into (2.4), we arrive at

$$
\sum_{k \in K_{d}}\left(M_{d} c\right)_{k} x^{k}=\lambda \sum_{k \in K_{d}} c_{k} x^{k}+\mathbf{p}_{d-1}(x)
$$

for some $\mathbf{p}_{d-1} \in \mathcal{P}_{d-1}$. Therefore, $M_{d} c=\lambda c$, i.e. $\lambda$ is an eigenvalue of $M_{d}$.

(2) On the other hand, if $\lambda$ is an eigenvalue of $M_{d}$, then there exists $c \in \mathbb{R}^{K_{d}} \backslash\{0\}$ such that $M_{d} c=\lambda c$. Let

$$
f(x)=\sum_{k \in K_{d}} c_{k} x^{k}-\pi_{d-1} \sum_{k \in K_{d}} c_{k} x^{k} .
$$

It follows from $M_{d} c=\lambda c$ and (2.5) that

$$
L_{\alpha}^{(N)} f=\tilde{\mathbf{p}}_{d-1}-\lambda f
$$

holds for some $\tilde{\mathbf{p}}_{d-1} \in \mathcal{P}_{d-1}$. Since $f \in \mathcal{Q}_{d}$ which is orthogonal to $\mathcal{P}_{d-1}$, this and (2.2) implies

$$
L_{\alpha}^{(N)} f=\left(1-\pi_{d-1}\right) L_{\alpha}^{(N)} f=-\lambda\left(1-\pi_{d-1}\right) f=-\lambda f .
$$


So, $\lambda$ is an eigenvalue of $L_{\alpha}^{(N)}$ on $\mathcal{Q}_{d}$.

(3) Finally, since eigenvalues of $-L_{\alpha}^{(N)}$ are nonnegative, (2) implies that eigenvalues of $\tilde{M}_{d}:=M_{d}-d \alpha_{N+1} I_{K_{d} \times K_{d}}$ is larger than or equal to $-d \alpha_{N+1}$. On the other hand, from the definition of $M_{d}$ we see that $\tilde{M}_{d}$ does not depend on $\alpha_{N+1}$. So, letting $\alpha_{N+1} \downarrow 0$ and noting that $M_{d} \geq 0$, we conclude that eigenvalues of $\tilde{M}_{d}$ are non-negative. Therefore, eigenvalues of $M_{d}$ are larger than or equal to $d \alpha_{N+1}$. Combining this with (1) we obtain $-\left.L_{\alpha}^{(N)}\right|_{\mathcal{Q}_{d}} \geq\left(d \alpha_{N+1}\right) I_{\mathcal{Q}_{d}}$.

Proposition 2.2. For $N \geq 2$, $\operatorname{gap}\left(L_{\alpha}^{(N)}\right)=\alpha_{N+1}$.

Proof: By Lemma 2.1, it suffices to prove that the smallest eigenvalue of $-\left.L_{\alpha}^{(N)}\right|_{\mathcal{Q}_{1}}$ is $\alpha_{N+1}$. To this end, we take $\theta_{i}=\left(\theta_{i j}\right)_{1 \leq j \leq N} \in \mathbb{R}^{N}(1 \leq i \leq N-1)$ such that

$$
\sum_{k=1}^{N} \theta_{i k} \alpha_{k}=0, \quad \sum_{k=1}^{N} \theta_{i k} \theta_{j k} \alpha_{k}=\delta_{i j}, \quad 1 \leq i, j \leq N-1 .
$$

So, $\left\{\theta_{i}\right\}_{i=1}^{N-1}$ is a basis of $\mathbb{R}^{N-1}$. Let

$$
\begin{aligned}
& u_{i}(x)=\sum_{j=1}^{N} \theta_{i j} x_{j}, \quad 1 \leq i \leq N-1 \\
& u_{N}(x)=\sum_{k=1}^{N} x_{k}-\frac{\tilde{\alpha}}{|\alpha|_{1}}, \quad \tilde{\alpha}:=|\alpha|_{1}-\alpha_{N+1}=\sum_{k=1}^{N} \alpha_{k} .
\end{aligned}
$$

We intend to prove that $\left\{u_{i}\right\}_{1 \leq i \leq N}$ is an orthogonal basis of $\mathcal{Q}_{1}$ with respect to the inner product $\langle f, g\rangle_{\alpha}^{(N)}:=\mu_{\alpha}^{(N)}(f g)=\int_{\Delta^{(N)}} f g \mathrm{~d} \mu_{\alpha}^{(N)}$, and $L_{\alpha}^{(N)} u_{N}=-|\alpha|_{1} u_{N}$ while $L_{\alpha}^{(N)} u_{i}=-\alpha_{N+1} u_{i}$ for $1 \leq i \leq N-1$. Thus, the smallest eigenvalue of $-\left.L_{\alpha}^{(N)}\right|_{\mathcal{Q}_{1}}$ is $\alpha_{N+1}$.

It is easy to see that

$$
\begin{aligned}
& \mu_{\alpha}^{(N)}\left(x_{i}\right):=\int_{\Delta^{(N)}} x_{i} \mu_{\alpha}^{(N)}(\mathrm{d} x)=\frac{\Gamma(\bar{\alpha}) \Gamma\left(\alpha_{i}+1\right)}{\Gamma\left(|\alpha|_{1}+1\right) \Gamma\left(\alpha_{i}\right)}=\frac{\alpha_{i}}{|\alpha|_{1}}, \\
& \mu_{\alpha}^{(N)}\left(x_{i}^{2}\right)=\frac{\Gamma(\bar{\alpha}) \Gamma\left(\alpha_{i}+2\right)}{\Gamma\left(|\alpha|_{1}+2\right) \Gamma\left(\alpha_{i}\right)}=\frac{\alpha_{i}\left(\alpha_{i}+1\right)}{|\alpha|_{1}\left(|\alpha|_{1}+1\right)}, \quad 1 \leq i \leq N-1 \\
& \mu_{\alpha}^{(N)}\left(x_{i} x_{j}\right)=\frac{\Gamma(\bar{\alpha}) \Gamma\left(\alpha_{i}+1\right) \Gamma\left(\alpha_{j}+1\right)}{\Gamma\left(|\alpha|_{1}+2\right) \Gamma\left(\alpha_{i}\right) \Gamma\left(\alpha_{j}\right)}=\frac{\alpha_{i} \alpha_{j}}{|\alpha|_{1}\left(|\alpha|_{1}+1\right)}, \quad 1 \leq i \neq j \leq N-1 .
\end{aligned}
$$

Then

$$
\begin{aligned}
& \mu_{\alpha}^{(N)}\left(u_{i}\right)=\frac{1}{|\alpha|_{1}} \sum_{k=1}^{N} \theta_{i k} \alpha_{k}=0, \quad 1 \leq i \leq N-1 \\
& \mu_{\alpha, \lambda}^{(N)}\left(u_{N}\right)=\sum_{i=1}^{N} \frac{\alpha_{i}}{|\alpha|_{1}}-\frac{\tilde{\alpha}}{|\alpha|_{1}}=0 .
\end{aligned}
$$

So, $\left\{u_{i}\right\}_{1 \leq i \leq N} \subset \mathcal{Q}_{1}$. Moreover, for $1 \leq i \neq j \leq N-1$,

$$
\begin{aligned}
& \mu_{\alpha}^{(N)}\left(u_{i} u_{j}\right)=\frac{1}{|\alpha|_{1}\left(|\alpha|_{1}+1\right)}\left(\sum_{1 \leq k \leq N} \theta_{i k} \theta_{j k} \alpha_{k}\left(\alpha_{k}+1\right)+\sum_{1 \leq k \neq l \leq N} \theta_{i k} \theta_{j l} \alpha_{k} \alpha_{l}\right) \\
& =\frac{1}{|\alpha|_{1}\left(|\alpha|_{1}+1\right)}\left\{\left(\sum_{1 \leq k \leq N} \theta_{i k} \alpha_{k}\right) \sum_{1 \leq l \leq N} \theta_{j l} \alpha_{l}+\sum_{1 \leq k \leq N} \theta_{i k} \theta_{j k} \alpha_{k}\right\}=0,
\end{aligned}
$$


and for any $1 \leq i \leq N-1$,

$$
\begin{aligned}
& \mu_{\alpha}^{(N)}\left(u_{i} u_{N}\right)=\sum_{1 \leq k, j \leq N} \theta_{i j} \mu\left(x_{j} x_{k}\right) \\
& =\frac{1}{|\alpha|_{1}\left(|\alpha|_{1}+1\right)} \sum_{1 \leq j \leq N} \theta_{i j} \alpha_{j}\left(\alpha_{j}+1\right)+\frac{1}{|\alpha|_{1}\left(|\alpha|_{1}+1\right)} \sum_{1 \leq k \neq j \leq N} \theta_{i j} \alpha_{j} \alpha_{k} \\
& =\frac{1}{|\alpha|_{1}\left(|\alpha|_{1}+1\right)} \sum_{1 \leq k, j \leq N} \theta_{i j} \alpha_{j} \alpha_{k}+\frac{1}{|\alpha|_{1}\left(|\alpha|_{1}+1\right)} \sum_{1 \leq j \leq N} \theta_{i j} \alpha_{j}=0 .
\end{aligned}
$$

Since $\left\{\theta_{i}\right\}_{i=1}^{N-1}$ is a basis of $\mathbb{R}^{N-1}$, we have

$$
\operatorname{dim} \operatorname{span}\left\{u_{i}: 1 \leq i \leq n-1\right\}=N-1=\operatorname{dim} \mathcal{Q}_{1} .
$$

In conclusion, $\left\{u_{i}\right\}_{1 \leq i \leq N}$ is an orthogonal basis of $\mathcal{Q}_{1}$.

Finally, we have

$$
L_{\alpha}^{(N)} u_{i}(x)=\sum_{j=1}^{N}\left(\alpha_{j} x_{N+1}-\alpha_{N+1} x_{j}\right) \theta_{i j}=-\alpha_{N+1} u_{i}, \quad 1 \leq i \leq N-1,
$$

and

$$
L_{\alpha}^{(N)} u_{N}(x)=\sum_{j=1}^{N}\left(\alpha_{j} x_{N+1}-\alpha_{N+1} x_{j}\right)=-|\alpha|_{1} \sum_{j=1}^{N} x_{j}+\sum_{j=1}^{N} \alpha_{j}=-|\alpha|_{1} u_{N}(x) .
$$

Therefore, the proof is finished.

\section{Proof of Theorem 1.1: the whole spectrum of $L_{\alpha}^{(N)}$}

For $d \in \mathbb{Z}_{+}$, let $\mathcal{H}_{d}$ be the space of homogeneous polynomials of total degree $d$ in the variables $x_{1}, \ldots, x_{N}$. Denote by $\tilde{\pi}_{d}$ the natural projection from $\mathcal{P}_{\infty}$ to $\mathcal{H}_{d}$ which only keeps the $d$-homogeneous part of a polynomial. Let $\bar{L}_{\alpha, d}^{(N)}=\left.\left(\tilde{\pi}_{d} L_{\alpha}^{(N)}\right)\right|_{\mathcal{H}_{d}}$ be the restriction of the operator $\tilde{\pi}_{d} L_{\alpha}^{(N)}$ to $\mathcal{H}_{d}$ and $-\Lambda_{d}$ denote its spectrum, seen as a multi-set (namely with multiplicities). From the above considerations, the spectrum $\Lambda$ of $-L_{\alpha}^{(N)}$ is equal to $\cup_{d \in \mathbb{Z}_{+}} \Lambda_{d}$, as a multi-set. We can write

$$
\bar{L}_{\alpha, d}^{(N)}=-|\cdot|{ }_{1} \tilde{L}_{\alpha, d}^{(N)}-\alpha_{N+1} \hat{L}_{\alpha, d}^{(N)},
$$

where $\tilde{L}_{\alpha, d}^{(N)}: \mathcal{H}_{d} \rightarrow \mathcal{H}_{d-1}$ and $\hat{L}_{\alpha, d}^{(N)}: \mathcal{H}_{d} \rightarrow \mathcal{H}_{d}$ are respectively the restriction to $\mathcal{H}_{d}$ of the operators

$$
\tilde{L}_{\alpha}^{(N)}:=\sum_{1 \leq n \leq N}\left(x_{n} \partial_{n}^{2}+\alpha_{n} \partial_{n}\right), \quad \hat{L}_{\alpha}^{(N)}:=\sum_{1 \leq n \leq N} x_{n} \partial_{n}
$$

under the projection $\tilde{\pi}_{d}$. The crucial point of the previous decomposition is that $\hat{L}_{\alpha, d}^{(N)}=d I_{\mathcal{H}_{d}}$. Denote by $\tilde{\Lambda}_{d}$ the spectrum of $|\cdot|{ }_{1} \tilde{L}_{\alpha, d}^{(N)}$, we thus have

$$
\Lambda_{d}=\tilde{\Lambda}_{d}+d \alpha_{N+1}
$$

Note that $\Lambda_{0}=\tilde{\Lambda}_{0}=\{0\}$. The next result enables to compute by iteration $\tilde{\Lambda}_{d}$ for all $d \in \mathbb{Z}_{+}$. 
Proposition 3.1. Let $\tilde{\alpha}=\sum_{i=1}^{N} \alpha_{i}$. For any $d \in \mathbb{Z}_{+}$, we have

$$
\tilde{\Lambda}_{d+1}=\left(2 d+\tilde{\alpha}+\tilde{\Lambda}_{d}\right) \cup\{0[C(N, d+1)-C(N, d)]\},
$$

where $\{0[l]\}$ is the multi-set with 0 repeated $l$ times, for $l \in \mathbb{Z}_{+}$(more generally $[l]$ will stand for the multiplicity $l)$, and where $C(N, d)$ is the dimension of $\mathcal{H}_{d}$, namely

$$
C(N, d)=\left(\begin{array}{c}
d+N-1 \\
d
\end{array}\right) \text {. }
$$

Proof: Consider $\lambda \in \tilde{\Lambda}_{d+1}$ and let $\varphi \in \mathcal{H}_{d+1}$ be an associated eigenvector (nonzero). We have

$$
|\cdot|{ }_{1} \tilde{L}_{\alpha, d+1}^{(N)} \varphi=\lambda \varphi .
$$

Since $\tilde{L}_{\alpha, d+1}^{(N)} \varphi \in \mathcal{H}_{d}$, there are two possibilities: either $\lambda=0$, or $\varphi=|\cdot|{ }_{1} \psi$ for some $\psi \in \mathcal{H}_{d}$ such that

$$
\tilde{L}_{\alpha, d+1}^{(N)}\left(|\cdot|{ }_{1} \psi\right)=\lambda \psi
$$

We consider the latter situation, since the former case leads to the multi-set $\{0[C(N, d+1)-C(N, d)]\}$.

We compute at point $x$ that

$$
\begin{aligned}
\tilde{L}_{\alpha, d+1}^{(N)}\left(|\cdot|{ }_{1} \psi\right) & =|x|_{1} \tilde{L}_{\alpha}^{(N)} \psi+\psi \tilde{L}_{\alpha}^{(N)}|\cdot|_{1}+2 \sum_{1 \leq n \leq N} x_{n} \partial_{n} \psi \\
& =|x|_{1} \tilde{L}_{\alpha, d}^{(N)} \psi+\psi \sum_{1 \leq n \leq N} \alpha_{n}+2 \sum_{1 \leq n \leq N} x_{n} \partial_{n} \psi \\
& =|x|_{1} \tilde{L}_{\alpha, d}^{(N)} \psi+(\tilde{\alpha}+2 d) \psi .
\end{aligned}
$$

So, it follows from (3.1) that $\lambda-\tilde{\alpha}-2 d$ is an eigenvalue of the operator $|\cdot|_{1} \tilde{L}_{\alpha, d}^{(N)}$, namely belongs to $\tilde{\Lambda}_{d}$. Thus,

$$
\tilde{\Lambda}_{d+1} \subset\left(2 d+\tilde{\alpha}+\tilde{\Lambda}_{d}\right) \cup\{0[C(N, d+1)-C(N, d)]\} .
$$

On the other hand, if $\lambda^{\prime} \in \tilde{\Lambda}_{d}$ then $|\cdot|_{1} \tilde{L}_{\alpha, d}^{(N)} \psi=\lambda^{\prime} \psi$ for some $0 \neq \psi \in \mathcal{H}_{d}$. Then (3.2) implies

$$
\tilde{L}_{\alpha, d+1}^{(N)}\left(|\cdot|{ }_{1} \psi\right)=|\cdot|{ }_{1} \tilde{L}_{\alpha, d}^{(N)} \psi+(\tilde{\alpha}+2 d) \psi=\left(\lambda^{\prime}+\tilde{\alpha}+2 d\right) \psi .
$$

Therefore, $\lambda^{\prime}+\tilde{\alpha}+2 d \in \tilde{\Lambda}_{d+1}$; that is, $\tilde{\Lambda}_{d+1} \supset\left(2 d+\tilde{\alpha}+\tilde{\Lambda}_{d}\right)$. Then the proof is finished.

Proof of Theorem 1.1: The previous arguments amount to an iterative construction of the eigenvectors: for any $d \in \mathbb{Z}_{+}$, let $\tilde{\mathcal{F}}_{d}$ be the set of eigenvectors of $|\cdot|_{1} \tilde{L}_{\alpha, d}^{(N)}$ and $\mathcal{G}_{d}$ be the kernel of $\tilde{L}_{\alpha, d}^{(N)}$. Then we have

$$
\forall d \in \mathbb{Z}_{+}, \quad \tilde{\mathcal{F}}_{d+1}=\mathcal{G}_{d+1} \cup\left(|\cdot|_{1} \tilde{\mathcal{F}}_{d}\right) .
$$

Indeed, in the above proof, functions $\varphi \in \tilde{\mathcal{F}}_{d+1}$ of the form $\mid \cdot{ }_{1} \psi$ with $\psi \in \tilde{\mathcal{F}}_{d}$ are associated to eigenvalues of the form $\tilde{\alpha}+2 d+\lambda$, where $\lambda \in \tilde{\Lambda}_{d}$. From Lemma 2.1, we know that $\lambda \geq 0$, so that $\tilde{\alpha}+2 d+\lambda>0$ and $\varphi$ does not belong to the kernel of $\tilde{L}_{d+1}^{(N)}$. Conversely, we have seen that all the other eigenvectors belong to the kernel of $\tilde{L}_{d+1}^{(N)}$. Thus we get the following characterization of the kernel of $\tilde{L}_{\alpha, d}^{(N)}$ : it consists exactly into the eigenvectors of $\tilde{L}_{\alpha, d}^{(N)}$ which don't admit $|\cdot|_{1}$ as a factor. 
Note that $\tilde{\mathcal{F}}_{d}$ is also the set of eigenvectors of $L_{\alpha, d}^{(N)}$. To get the eigenvectors of our initial operator $L_{\alpha}^{(N)}$, we construct by iteration on $d \in \mathbb{Z}_{+}$the following subsets $\mathcal{F}_{d}$ of $\mathcal{P}_{d}$. First we take $\mathcal{F}_{0}:=\tilde{\mathcal{F}}_{0}=\mathcal{P}_{0}$. Next, if $\mathcal{F}_{d}$ has been constructed, then for any $f \in \tilde{\mathcal{F}}_{d+1}$, there exists a unique $g_{f} \in \mathcal{P}_{d}$ such that $f+g_{f}$ is orthogonal to $\mathcal{P}_{d}$ in $\Lambda^{2}(\mu)$. Then we define

$$
\mathcal{F}_{d+1}:=\left\{f+g_{f}: f \in \tilde{\mathcal{F}}_{d+1}\right\} .
$$

The set of eigenvectors of $L_{\alpha}^{(N)}$ is $\cup_{d \in \mathbb{Z}_{+}} \mathcal{F}_{d}$.

From Proposition 3.1, $K: \mathcal{K} \rightarrow \Lambda$ is surjective. It is truly one-to-one, if and only if $1, \tilde{\alpha}$ and $\alpha_{N+1}$ are independent when $\mathbb{R}$ is seen as a vector space over $\mathbb{Q}$. Let us call this situation generical over the choice of the parameters $\alpha:=\left(\alpha_{n}\right)_{1 \leq n \leq N+1}$. Moreover, the multiplicity of an eigenvalue $\lambda \in \Lambda$ is given by

$$
\sum_{k \in K^{-1}(\lambda)} D(k)
$$

Therefore, $\Lambda=\{K(k)[D(k)]: k \in \mathcal{K}\}$.

\section{Proofs of Theorems 1.2 and 1.3}

To prove the first assertion, let $W$ be the $L^{1}$-Wasserstein distance induced by $\rho(x, y):=|x-y|_{1}$ on $\mathcal{P}\left(\Delta^{(\infty)}\right)$, the set of all probability measures on $\Delta^{(\infty)}$. That is, for any $\mu, \nu \in \mathcal{P}\left(\Delta^{(\infty)}\right)$,

$$
W(\mu, \nu):=\inf _{\pi \in \mathcal{C}(\mu, \nu)} \int_{\Delta(\infty) \times \Delta(\infty)}|x-y|_{1} \pi(\mathrm{d} x, \mathrm{~d} y),
$$

where $\mathcal{C}(\mu, \nu)$ is the set of all couplings for $\mu$ and $\nu$; i.e. $\pi \in \mathcal{C}(\mu, \nu)$ if and only if it is a probability measure on $\Delta^{(\infty)} \times \Delta^{(\infty)}$ such that

$$
\pi\left(\mathrm{d} x \times \Delta^{(\infty)}\right)=\mu(\mathrm{d} x), \quad \pi\left(\Delta^{(\infty)} \times \mathrm{d} y\right)=\nu(\mathrm{d} y) .
$$

It is well known that the metric $W$ is complete and induces the weak topology on $\mathcal{P}\left(\Delta^{(\infty)}\right)$, see e.g. Chen (1992, Theorems 5.4 and 5.6). So, for the proof of Theorem 1.2 we only need to show that $\left\{\mu_{\alpha, \alpha_{\infty}}^{(n)}\right\}_{n \geq 1}$ is $W$-Cauchy sequence.

Proof of Theorem 1.2: Let $\mathcal{L}\{\xi\}$ denote the law of a random variable $\xi$.

(1) To prove that $\left\{\mu_{\alpha, \alpha_{\infty}}^{(n)}\right\}_{n \geq 1}$ is a $W$-Cauchy sequence, we use the partition property of the Dirichlet distribution mentioned in Section 1. For any $n>m \geq$ 1 , let $\left(X_{1}^{(n)}, \cdots, X_{n+1}^{(n)}\right)$ have law $\tilde{\mu}_{\alpha^{(n)}}^{(n+1)}$. By the partition property, $\tilde{\mu}_{\alpha^{(m)}}^{(m+1)}=$ $\mathcal{L}\left\{\left(X_{1}^{(n)}, \cdots, X_{m-1}^{(n)}, \sum_{i=m}^{n} X_{i}^{(n)}, X_{n+1}^{(n)}\right)\right\}$. So,

$$
\mu_{\alpha^{(m)}}^{(m)}=\mathcal{L}\left\{\left(X_{1}^{(n)}, \cdots, X_{m-1}^{(n)}, \sum_{i=m}^{n} X_{i}^{(n)}\right)\right\}, \quad \mu_{\alpha^{(n)}}^{(n)}=\mathcal{L}\left\{\left(X_{1}^{(n)}, \cdots, X_{n}^{(n)}\right)\right\} .
$$

Thus,

$$
\begin{aligned}
& \mu_{\alpha, \alpha_{\infty}}^{(m)}=\mathcal{L}\left\{\left(X_{1}^{(n)}, \cdots, X_{m-1}^{(n)}, \sum_{i=m}^{n} X_{i}^{(n)}, 0,0, \cdots\right)\right\}, \\
& \mu_{\alpha, \alpha_{\infty}}^{(n)}=\mathcal{L}\left\{\left(X_{1}^{(n)}, \cdots, X_{n}^{(n)}, 0,0, \cdots\right)\right\} .
\end{aligned}
$$


Then, by the definition of $W$ and noting that $|\alpha|_{1}<\infty$, we have

$$
\begin{aligned}
& \limsup _{m \rightarrow \infty} \sup _{n \geq m+1} W\left(\mu_{\alpha, \alpha_{\infty}}^{(m)}, \mu_{\alpha, \alpha_{\infty}}^{(n)}\right) \leq 2 \limsup _{m \rightarrow \infty} \sup _{n \geq m+1} \sum_{i=m+1}^{n} \mathbb{E}\left|X_{i}^{(n)}\right| \\
& \leq \limsup _{m \rightarrow \infty} \frac{2 \sum_{i=m+1}^{\infty} \alpha_{i}}{\alpha_{\infty}+\|\alpha\|_{1}}=0 .
\end{aligned}
$$

Therefore, $\left\{\mu_{\alpha, \alpha_{\infty}}^{(n)}\right\}_{n \geq 1}$ is a $W$-Cauchy sequence and the proof of the first assertion is finished.

(2) It suffices to prove

$$
\mathcal{E}_{\alpha, \alpha_{\infty}}^{(\infty)}(f, g)=-\int_{\Delta(\infty)}\left(f L_{\alpha, \alpha_{\infty}}^{(\infty)} g\right) \mathrm{d} \mu_{\alpha, \alpha_{\infty}}^{(\infty)}, \quad f, g \in \mathcal{F} C^{2}
$$

For any $f, g \in \mathcal{F} C^{2}$, there exist $m \in \mathbb{N}$ and $f_{m}, g_{m} \in C^{2}\left(\mathbb{R}^{m}\right)$ such that

$$
f(x)=f_{m}\left(x_{1}, \cdots, x_{m}\right), \quad g(x)=g_{m}\left(x_{1}, \cdots, x_{m}\right), \quad x \in \Delta^{(\infty)} .
$$

So, by the definition of $\mu_{\alpha, \alpha_{\infty}}^{(n)}$ and using (1.3), we have

$$
-\int_{\Delta(\infty)}\left(f L_{\alpha^{(n)}}^{(n)} g\right) \mathrm{d} \mu_{\alpha, \alpha_{\infty}}^{(n)}=\int_{\Delta(\infty)}\left\{\left(1-|x|_{1}\right) \sum_{i=1}^{m} x_{i}\left(\partial_{i} f\right)\left(\partial_{i} g\right)\right\} \mathrm{d} \mu_{\alpha, \alpha_{\infty}}^{(n)} .
$$

Since $\mu_{\alpha, \alpha_{\infty}}^{(n)} \rightarrow \mu_{\alpha, \alpha_{\infty}}^{(\infty)}$ weakly, and it is easy to see that

$$
\begin{aligned}
& \lim _{n \rightarrow \infty} \sup _{x \in \Delta^{(\infty)}}\left|f L_{\alpha^{(n)}}^{(n)} g-f L_{\alpha, \alpha_{\infty}} g\right|(x)=0, \\
& \lim _{n \rightarrow \infty} \sup _{x \in \Delta^{(\infty)}}\left|\left(1-|x|_{1}\right) \sum_{i=1}^{m} x_{i}\left(\partial_{i} f\right)\left(\partial_{i} g\right)-\left(1-\sum_{i=1}^{\infty} x_{i}\right) \sum_{i=1}^{m} x_{i}\left(\partial_{i} f\right)\left(\partial_{i} g\right)\right|=0,
\end{aligned}
$$

by letting $n \rightarrow \infty$ in (4.2) we prove (4.1).

(3) Finally, as was shown in (2) that the desired Poincaré inequality follows by applying Theorem 1.1 to $\mu_{\alpha^{(n)}}^{(n)}$ on $\Delta^{(n)}$ then letting $n \rightarrow \infty$. So, $\operatorname{gap}\left(L_{\alpha, \alpha_{\infty}}^{(\infty)}\right) \geq \alpha_{\infty}$. On the other hand, let

$$
u(x)=\alpha_{2} x_{1}-\alpha_{1} x_{2}, \quad x \in \Delta^{(\infty)} .
$$

We have

$$
\begin{aligned}
L_{\alpha, \alpha_{\infty}}^{(\infty)} u(x) & =\left\{\alpha_{1}\left(1-|x|_{1}\right)-\alpha_{\infty} x_{1}\right\} \alpha_{2}-\left\{\alpha_{2}\left(1-|x|_{1}\right)-\alpha_{\infty} x_{2}\right\} \alpha_{1} \\
& =-\alpha_{\infty} u(x), \quad \text { for } x \in \Delta^{(\infty)}
\end{aligned}
$$

This implies $\operatorname{gap}\left(L_{\alpha, \alpha_{\infty}}^{(\infty)}\right) \leq \alpha_{\infty}$. In conclusion, we have $\operatorname{gap}\left(L_{\alpha, \alpha_{\infty}}^{(\infty)}\right)=\alpha_{\infty}$.

Proof of Theorem 1.3: (a) For the first assertion, we only need to prove that $\left\{P_{x, T}^{(n)}\right\}_{n \geq 1}$ is a Cauchy sequence with respect to the $L^{1}$-Wasserstein distance

$$
W_{T}\left(P, P^{\prime}\right):=\inf _{\Pi \in \mathcal{C}\left(P, P^{\prime}\right)} \int_{\Omega_{T} \times \Omega_{T}}\|\xi-\eta\|_{1, \infty} \Pi(\mathrm{d} \xi, \mathrm{d} \eta) .
$$

To this end, for any $n>m \geq 2$, we construct a coupling of $P_{x, T}^{(n)}$ and $P_{x, T}^{(m)}$ as follows. 
Firstly, let $\left(X_{i}^{(n)}(t)\right)_{1 \leq i \leq n}$ solve the following SDE with $X_{0}^{(n)}=x^{(n)}$ :

$$
\begin{aligned}
\mathrm{d} X_{i}^{(n)}(t)= & {\left[\alpha_{i}\left(1-\left|X^{(n)}(t)\right|_{1}\right)-\alpha_{\infty} X_{i}^{(n)}(t)\right] \mathrm{d} t } \\
& +\sqrt{2\left(1-\left|X^{(n)}(t)\right|_{1}\right) X_{i}^{(n)}(t)} \mathrm{d} B_{i}(t), 1 \leq i \leq n-1 ; \\
\mathrm{d} X_{n}^{(n)}(t)= & {\left[\sum_{j=n}^{\infty} \alpha_{j}\left(1-\left|X^{(n)}(t)\right|_{1}\right)-\alpha_{\infty} X_{n}^{(n)}(t)\right] \mathrm{d} t } \\
& +\sqrt{2\left(1-\left|x^{(n)}(t)\right|_{1}\right) X_{n}^{(n)}(t)} \mathrm{d} B_{n}(t), \quad t \in[0, T],
\end{aligned}
$$

where $\left(B_{i}(t)_{1 \leq i \leq n}\right.$ are independent one-dimensional Brownian motions. Then $P_{x, T}^{(n)}$ is the distribution of $\left(X^{(n)}(t)\right)_{t \in[0, T]}$.

Next, let

$$
X_{i}^{(m)}(t)=X_{i}^{(n)}(t) \text { for } 1 \leq i \leq m-1, \text { and } X_{m}^{(m)}(t)=\sum_{j=m^{n}} X_{j}^{(n)}(t), \quad t \in[0, T] .
$$

Then $X^{(m)}(0)=x^{(m)}$ and by (4.3),

$$
\begin{aligned}
\mathrm{d} X_{i}^{(m)}(t)= & {\left[\alpha_{i}\left(1-\left|X^{(m)}(t)\right|_{1}\right)-\alpha_{\infty} X_{i}^{(m)}(t)\right] \mathrm{d} t } \\
& +\sqrt{2\left(1-\left|x^{(m)}(t)\right|_{1}\right) X_{i}^{(m)}(t)} \mathrm{d} B_{i}(t), 1 \leq i \leq m-1 ; \\
\mathrm{d} X_{m}^{(m)}(t)= & {\left[\sum_{j=m}^{\infty} \alpha_{j}\left(1-\left|X^{(m)}(t)\right|_{1}\right)-\alpha_{\infty} X_{m}^{(m)}(t)\right] \mathrm{d} t } \\
& +\sqrt{2\left(1-\left|x^{(m)}(t)\right|_{1}\right) X_{m}^{(m)}(t)} \mathrm{d} \tilde{B}_{m}(t), \quad t \in[0, T],
\end{aligned}
$$

where $\mathrm{d} \tilde{B}_{m}(t):=\frac{1}{\sqrt{X_{m}^{(m)}(t)}} \sum_{i=m}^{n} \sqrt{X_{i}^{(n)}(t)} \mathrm{d} B_{i}(t)$ is a one-dimensional Brownian motion independent of $\left(B_{i}(t)\right)_{1 \leq i \leq m-1}$. Therefore, $\left(X^{(m)}(t)\right)_{t \in[0, T]}$ has law $P_{x, T}^{(m)}$.

Now, by (4.4) and the definition of $W_{T}$, we have

$$
W_{T}\left(P_{x, T}^{(n)}, P_{x, T}^{(m)}\right) \leq \mathbb{E} \sup _{t \in[0, T]}\left|X^{(m)}(t)-X^{(n)}(t)\right|_{1}=\mathbb{E} \sup _{t \in[0, T]} \sum_{j=m+1}^{n} X_{j}^{(n)}(t) .
$$

Let $Z(t)=\sum_{j=m+1}^{n} X_{j}^{(n)}(t)$. By (4.3) we have

$$
\mathrm{d} Z(t) \leq\left(\sum_{j=m+1}^{\infty} \alpha_{j}\right) \mathrm{d} t+\sum_{j=m+1}^{n} \sqrt{s\left(1-\left|X^{(n)}(t)\right|_{1}\right) X_{i}^{(n)}(t)} \mathrm{d} B_{i}(t) .
$$

So,

$$
\begin{aligned}
Z(t) & \leq \sum_{j=1+m}^{\infty}\left(x_{j}+t \alpha_{j}\right)+\sum_{j=m+1}^{n} \int_{0}^{t} \sqrt{s\left(1-\left|X^{(n)}(s)\right|_{1}\right) X_{i}^{(n)}(s)} \mathrm{d} B_{i}(s) \\
& =: \bar{Z}(t), \quad t \in[0, T] .
\end{aligned}
$$


Since $Z(t) \geq 0, \bar{Z}(t)$ is a nonnegative submartingale. Then by Kolmogorov's inequality,

$\mathbb{P}\left(\sup _{t \in[0, T]} Z(t) \geq \lambda\right) \leq \mathbb{P}\left(\sup _{t \in[0, T]} \bar{Z}(t) \geq \lambda\right) \leq \frac{1}{\lambda} \mathbb{E} \bar{Z}(T)=\frac{1}{\lambda} \sum_{j=m+1}^{\infty}\left(x_{j}+\alpha_{j} T\right), \lambda>0$.

Since $Z(t) \leq 1$, this implies

$$
\mathbb{E} \sup _{t \in[0, T]} Z(t) \leq \lambda+\mathbb{P}\left(\sup _{t \in[0, T]} Z(t) \geq \lambda\right) \leq \lambda+\frac{1}{\lambda} \sum_{j=m+1}^{\infty}\left(x_{j}+\alpha_{j} T\right), \quad \lambda>0 .
$$

Taking $\lambda=\sqrt{\sum_{j=m+1}^{\infty}\left(x_{j}+\alpha_{j} T\right)}$, and combining with (4.5), we obtain

$$
\lim _{m \rightarrow \infty} \sup _{n \geq m+1} W_{T}\left(P_{x, T}^{(n)}, P_{x, T}^{(m)}\right) \leq 2 \lim _{m \rightarrow \infty} \sqrt{\sum_{j=m+1}^{\infty}\left(x_{j}+\alpha_{j} T\right)}=0 .
$$

Therefore, the first assertion is proved.

(b) Let $f \in \mathcal{F} C^{2}$. We have $f(x)=f\left(x_{1}, \cdots, x_{m}\right)$ for some $m \geq 1$ and $f \in$ $C^{2}\left(\Delta^{(m)}\right)$. For the coordinate process $X(t)$, define

$$
M^{(n)}(t)=f(X(t))-\int_{0}^{t} L_{\alpha, \alpha_{\infty}}^{(n)} f(X(s)) \mathrm{d} s, \quad n \geq m, t \in[0, T] .
$$

Then $\left(M_{t}^{(n)}\right)_{t \in[0, T]}$ is a $P_{x, T}^{(n)}$-martingale; that is, for any $0<s<t \leq T$, and any bounded Lipschitz continuous function $g$ on $\Omega_{T}$ measurable with respect to $\mathcal{F}_{s}$,

$$
\int_{\Omega_{T}} M^{(n)}(t)(\omega) g(\omega) \mathrm{d} P_{x, T}^{(n)}=\int_{\Omega_{T}} M^{(n)}(s)(\omega) g(\omega) \mathrm{d} P_{x, T}^{(n)}
$$

We intend to prove the same equality for $P_{x, T}^{(\infty)}$ and

$$
M^{(\infty)}(t):=f(X(t))-\int_{0}^{t} L_{\alpha, \alpha_{\infty}}^{(\infty)} f(X(s)) \mathrm{d} s, t \in[0, T] .
$$

By an approximation argument, we may and do assume that $f \in C_{b}^{3}\left(\Delta^{(m)}\right)$. In this case, $M^{(n)}(t)$ is bounded and Lipschitz on $\Omega_{T}$ uniformly in $n \geq m$ and $t \in[0, T]$. Since $g$ is bounded and Lipschitz on $\Omega_{T}$ as well, there exists a constant $C>0$ such that

$$
\left|\left(M^{(n)}(t) g\right)(\xi)-\left(M^{(n)} g\right)(t)(\eta)\right| \leq C\|\xi-\eta\|_{1, \infty}, \quad n \geq m, \xi, \eta \in \Omega_{T}, t \in[0, T] .
$$

Therefore,

$$
\left|\int_{\Omega_{T}} M^{(n)}(t) g \mathrm{~d} P_{x, T}^{(n)}-\int_{\Omega_{T}} M^{(n)}(t) g \mathrm{~d} P_{x, T}^{(\infty)}\right| \leq C W_{T}\left(P_{x, T}^{(n)}, P_{x, T}^{(\infty)}\right), \quad n \geq m, t \in[0, T] .
$$

Combining this with (4.6), $\lim _{n \rightarrow \infty} W_{T}\left(P_{x, T}^{(n)}, P_{x, T}^{(\infty)}\right)=0, \lim _{n \rightarrow \infty} M^{(n)}=M^{(\infty)}$ and noting that $\left\{M^{(n)} g\right\}_{n \geq m}$ are uniformly bounded, we conclude that

$$
\begin{aligned}
& \left|\int_{\Omega_{T}}\left[M^{(\infty)}(t)-M^{(\infty)}(s)\right] g \mathrm{~d} P_{x, T}^{(\infty)}\right| \\
& =\lim _{n \rightarrow \infty}\left|\int_{\Omega_{T}}\left[M^{(n)}(t)-M^{(n)}(s)\right] g \mathrm{~d} P_{x, T}^{(\infty)}\right| \\
& \leq 2 C \limsup _{n \rightarrow \infty} W_{T}\left(P_{x, T}^{(n)}, P_{x, T}^{(\infty)}\right)=0 .
\end{aligned}
$$


Then the proof is finished.

\section{A Discrete Model}

For any $N \geq 1, M \geq N+1$, consider a population of $M$ individuals of $N+1$ different types. Divide the population into two groups: group I of types $1, \ldots, N$ and group II of type $N+1$. Focusing on group I and treat group II as outsiders or external sources. Initially the number of type $i$ individuals is $m_{i}, i=1, \ldots, N+1$. The group I evolves as follows: a type $i$ individual independent of all others will wait for an exponential time at rate $\alpha_{N+1}$ and at the end of the waiting emigrates to the outside becoming type $N+1$; an outsider will independently wait for an exponential time with rate $\alpha_{i}$ and immigrate to group I becoming type $i$; in addition to emigration and immigration, each couple between a type I and a type II waits for an exponential time with rate 2 and when the clock rings, either the group I individual moves out becoming an outsider or the group II individual moves in becoming the type of the selected individual in group I.

Let $X(t)=M^{-1}\left(M_{1}(t), \ldots, M_{N}(t)\right)$ denote the relative frequencies of individuals of different types in group $I$ among the whole population at time $t$. For $\alpha \in(0, \infty)^{N+1}$, we construct $X(t)$ as a multivariate Markov chain with generator

$$
\begin{aligned}
& \mathcal{A}_{M, \alpha}^{(N)} f(x) \\
& =M \sum_{i=1}^{N}\left\{\alpha_{N+1} x_{i}\left[f\left(x-\frac{e_{i}}{M}\right)-f(x)\right]+\alpha_{i}\left(1-|x|_{1}\right)\left[f\left(x+\frac{e_{i}}{M}\right)-f(x)\right]\right\} \\
& +M^{2} \sum_{i=1}^{N}\left(1-|x|_{1}\right) x_{i}\left\{f\left(x-\frac{e_{i}}{M}\right)+f\left(x+\frac{e_{i}}{M}\right)-2 f(x)\right\}, \quad f \in C^{2}\left(\Delta^{(N)}\right)
\end{aligned}
$$

for $x \in \Delta_{M}^{(N)}:=\left\{x \in \frac{1}{M} \mathbb{Z}_{+}^{N}:|x|_{1}=\sum_{i=1}^{N} x_{i} \leq 1\right\}$, where $e_{i}$ is the unit vector in the $i$ th direction. Letting $M \rightarrow \infty$ and $x \rightarrow y \in \Delta^{(N)}$, one gets $\mathcal{A}_{M, \alpha}^{(N)} f(x) \rightarrow L_{\alpha}^{(N)} f(y)$.

We will see that the finite Markov chain generated by $\mathcal{A}_{M, \alpha}^{(N)}$ on $\Delta_{M}^{(N)}$ is reversible with respect to the probability measure $\mu_{M, \alpha}^{(N)}$ :

$$
\mu_{M, \alpha}^{(N)}(x):=\frac{\left[\alpha_{N+1}\right]_{M\left(1-|x|_{1}\right)}}{Z\left\{M\left(1-|x|_{1}\right)\right\} !} \prod_{i=1}^{N} \frac{\left[\alpha_{i}\right]_{M x_{i}}}{\left(M x_{i}\right) !}, \quad x \in \Delta_{M}^{(N)},
$$

where $[\alpha]_{m}:=\prod_{i=0}^{m-1}(\alpha+i)$ for $\alpha \geq 0$ and $m \geq 1,[\alpha]_{0}:=1$, and

$$
Z:=\sum_{x \in \Delta_{M}^{(N)}} \frac{\left[\alpha_{N+1}\right]_{M\left(1-|x|_{1}\right)}}{\left\{M\left(1-|x|_{1}\right)\right\} !} \prod_{i=1}^{N} \frac{\left[\alpha_{i}\right]_{M x_{i}}}{\left(M x_{i}\right) !}
$$

is the normalization. Moreover, for $N \geq 2, \mathcal{A}_{M, \alpha}^{(N)}$ has the same spectral gap $\alpha_{N+1}$ as $L_{\alpha}^{(N)}$.

Theorem 5.1. Let $N \geq 2$. The Markov chain generated by $\mathcal{A}_{M, \alpha}^{(N)}$ is irreducible and reversible with respect to $\mu_{M, \alpha}^{(N)}$. Moreover, $\mathcal{A}_{M, \alpha}^{(N)}$ has spectral gap $\alpha_{N+1}$ in $L^{2}\left(\mu_{M, \alpha}^{(N)}\right)$. 
Proof: (a) Denote $\gamma_{i}=\frac{e_{i}}{M}$ for $1 \leq i \leq N$. For any $x, y \in \Delta_{M}^{(N)}$, let

$$
q_{x, y}= \begin{cases}M x_{i} \alpha_{N+1}+M^{2} x_{i}\left(1-|x|_{1}\right), & \text { if } y=x-\gamma_{i}, 1 \leq i \leq N \\ \alpha_{i} M\left(1-|x|_{1}\right)+M^{2} x_{i}\left(1-|x|_{1}\right), & \text { if } y=x+\gamma_{i}, 1 \leq i \leq N \\ 0, & \text { otherwise }\end{cases}
$$

We have

$$
\mathcal{A}_{M, \alpha}^{(N)} f(x)=\sum_{y \in \Delta_{M}^{(N)}} q_{x y}\{f(y)-f(x)\}, \quad x \in \Delta_{M}^{(N)} .
$$

Since $q_{x, y}>0$ when $x, y \in \Delta_{M}^{(N)}$ with $y=x \pm \gamma_{i}$ for $1 \leq i \leq N$, and $\Delta_{M}^{(N)}$ is connected by the edges $x \rightarrow x \pm \gamma_{i}$, we see that the Markov chain is irreducible.

Next, it is well known that $\mathcal{A}_{M, \alpha}^{(N)}$ is symmetric in $L^{2}\left(\mu_{M, \alpha}^{(N)}\right)$ if and only if

$$
\mu_{M, \alpha}^{(N)}(x) q_{x, y}=\mu_{M, \alpha}^{(N)}(y) q_{y, x}, \quad x, y \in \Delta_{M}^{(N)} .
$$

To verify this condition, we only need to consider the following two situations.

(a1) $y=x+\gamma_{i}$ for some $1 \leq i \leq N$. In this case we have $M|x|_{1} \leq M-1$, and by the definition of $\mu_{M, \alpha}^{(N)}$,

$$
\frac{\mu_{M, \alpha}^{(N)}(y)}{\mu_{M, \alpha}^{(N)}(x)}=\frac{M\left(1-|x|_{1}\right)\left(\alpha_{i}+M x_{i}\right)}{\left(\alpha_{N+1}+M\left(1-|x|_{1}\right)-1\right)\left(M x_{i}+1\right)}=\frac{q_{x y}}{q_{y x}} .
$$

(a2) $y=x-\gamma_{i}$ for some $1 \leq i \leq N$. In this case we have $M x_{i} \geq 1$, and by the definition of $\mu_{M, \alpha}^{(N)}$,

$$
\frac{\mu_{M, \alpha}^{(N)}(y)}{\mu_{M, \alpha}^{(N)}(x)}=\frac{\left(\alpha_{N+1}+M\left(1-|x|_{1}\right)\right) M x_{i}}{\left(M\left(1-|x|_{1}\right)+1\right)\left(M x_{i}-1+\alpha_{i}\right)}=\frac{q_{x y}}{q_{y x}} .
$$

In conclusion, (5.1) holds and thus, $\mathcal{A}_{M, \alpha}^{(N)}$ is symmetric in $L^{2}\left(\mu_{M, \alpha}^{(N)}\right)$.

(b) For any $d \in \mathbb{Z}_{+}$, consider again $\mathcal{P}_{d}$ the space of all polynomials (in $N$ variables) whose total degree is less than or equal to $d$. For any $f \in \mathcal{P}_{d}$ and $1 \leq i \leq N, x \mapsto f\left(x-\gamma_{i}\right)-f(x)$ and $x \mapsto f\left(x+\gamma_{i}\right)-f(x)$ are polynomials belonging to $\mathcal{P}_{d-1}$, while $x \mapsto f\left(x-\gamma_{i}\right)+f\left(x+\gamma_{i}\right)-2 f(x)$ is a polynomial belonging to $\mathcal{P}_{d-2}$. From the definition of $\mathcal{A}_{M, \alpha}^{(N)}$, it follows that $\mathcal{P}_{d}$ is preserved by $\mathcal{A}_{M, \alpha}^{(N)}$. As in Section 2 , we consider for $d \in \mathbb{Z}_{+}$,

$$
\mathcal{Q}_{d}:=\left\{f \in \mathcal{P}_{d} \cap L^{2}\left(\mu_{M, \alpha}^{(N)}\right): \mu_{M, \alpha}^{(N)}[f g]=0, \forall g \in \mathcal{P}_{d-1}\right\}
$$

(with the convention $\mathcal{Q}_{0}=\mathcal{P}_{0}$ ). Note that for $d$ large enough, $\mathcal{Q}_{d}=\{0\}$, nevertheless, we still have

$$
L^{2}\left(\mu_{M, \alpha}^{(N)}\right)=\bigoplus_{d \in \mathbb{Z}_{+}} \mathcal{Q}_{d}
$$

and the $\mathcal{Q}_{d}$ are orthogonal. Furthermore by symmetry of $\mathcal{A}_{M, \alpha}^{(N)}$ in $L^{2}\left(\mu_{M, \alpha}^{(N)}\right)$, each of the $\mathcal{Q}_{d}$ is preserved by $\mathcal{A}_{M, \alpha}^{(N)}$. Thus it is sufficient to study the spectral decompositions of the restrictions of $\mathcal{A}_{M, \alpha}^{(N)}$ to the $\mathcal{Q}_{d}$. But this is exactly the same analysis as in Section 2, because there we only used the highest monomials. Indeed, note that for all $f \in \mathcal{Q}_{d}$ and $1 \leq i \leq N$,

$$
x \mapsto f\left(x-\gamma_{i}\right)-f(x)+\frac{\partial_{i} f(x)}{M}, \quad x \mapsto f\left(x+\gamma_{i}\right)-f(x)-\frac{\partial_{i} f(x)}{M}
$$


are polynomials belonging to $\mathcal{P}_{d-2}$, and

$$
x \mapsto f\left(x-\gamma_{i}\right)+f\left(x+\gamma_{i}\right)-2 f(x)-\frac{\partial_{i}^{2} f(x)}{M^{2}}
$$

belong to $\mathcal{P}_{d-3}$, where we set $\mathcal{P}_{k}=\{0\}$ if $k<0$. Thus, for any polynomial $f \in \mathcal{Q}_{d}$, the polynomials $\mathcal{A}_{M, \alpha}^{(N)} f$ and $L_{\alpha}^{(N)} f$ have the same highest order term (i.e. the term of degree $d$ ), so that these two operators have the same spectral gap.

Finally, we show that $\mu_{M, \alpha}^{(N)}$ converges weakly to $\mu_{\alpha}^{(N)}$ as $M \rightarrow \infty$.

Proposition 5.2. Under the topology on $\Delta^{(N)}$ induced by $|\cdot|_{1}, \mu_{M, \alpha}^{(N)}$ converges weakly to $\mu_{\alpha}^{(N)}$ as $M \rightarrow \infty$.

Proof: It suffices to prove that $\mu_{M, \alpha}^{(N)}(f) \rightarrow \mu_{\alpha}^{(N)}(f)$ for any polynomial $f$. We first consider $\mu(f)=0$, i.e. $f \in \mathcal{P}_{0, d}$ for some $d \geq 1$. Since $\left.L_{\alpha}^{(N)}\right|_{\mathcal{P}_{0, d}}$ is bounded with eigenvalues not larger than $-\alpha_{N+1}, L_{\alpha}^{(N)}$ is invertible on $\mathcal{P}_{0, d}$. So, there exists $g \in \mathcal{P}_{0, d}$ such that $f=L_{\alpha}^{(N)} g$. Noting that

$$
\lim _{M \rightarrow \infty}\left\|\mathcal{A}_{M, \alpha}^{(N)} g-L_{\alpha}^{(N)} g\right\|_{\infty}=0, \quad \mu_{\alpha, M}^{(N)}\left(\mathcal{A}_{M, \alpha}^{(N)} g\right)=0
$$

we obtain

$$
\lim _{M \rightarrow \infty}\left|\mu_{\alpha, M}^{(N)}(f)\right|=\lim _{M \rightarrow \infty}\left|\mu_{\alpha, M}^{(N)}\left(L_{\alpha}^{(N)} g\right)\right|=\lim _{M \rightarrow \infty}\left|\mu_{\alpha, M}^{(N)}\left(\mathcal{A}_{\alpha, M}^{(N)} g\right)\right|=0 .
$$

That is, $\lim _{M \rightarrow \infty} \mu_{\alpha, M}^{(N)}(f)=\mu_{\alpha}^{(N)}(f)$ holds for any polynomial $f$ with $\mu(f)=0$. In general, if $\mu(f) \neq 0$, by letting $\hat{f}=f-\mu(f)$ we obtain

$$
0=\lim _{M \rightarrow \infty} \mu_{\alpha, M}^{(N)}(\hat{f})=\lim _{M \rightarrow \infty} \mu_{\alpha, M}^{(N)}(f)-\mu(f) .
$$

Then the proof is finished.

\section{Acknowledgements}

The authors would like to thank an anonymous referee for helpful comments.

\section{References}

J. Bakosi and J. R. Ristorcelli. A stochastic diffusion process for the Dirichlet distribution. Int. J. Stoch. Anal. pages Art. ID 842981, 7 (2013). MR3042549.

D. Bakry and M. Émery. Hypercontractivité de semi-groupes de diffusion. $C$. $R$. Acad. Sci. Paris Sér. I Math. 299 (15), 775-778 (1984). MR772092.

D. Blackwell and J. B. MacQueen. Ferguson distributions via Pólya urn schemes. Ann. Statist. 1, 353-355 (1973). MR0362614.

M. F. Chen. From Markov chains to nonequilibrium particle systems. World Scientific Publishing Co., Inc., River Edge, NJ (1992). ISBN 981-02-0639-9. MR1168209.

R. J. Connor and J. E. Mosimann. Concepts of independence for proportions with a generalization of the Dirichlet distribution. J. Amer. Statist. Assoc. 64, 194-206 (1969). MR0240895. 
C. L. Epstein and R. Mazzeo. Wright-Fisher diffusion in one dimension. SIAM J. Math. Anal. 42 (2), 568-608 (2010). MR2607922.

C. L. Epstein and R. Mazzeo. Degenerate diffusion operators arising in population biology, volume 185 of Annals of Mathematics Studies. Princeton University Press, Princeton, NJ (2013). ISBN 978-0-691-15715-3. MR3202406.

S. N. Ethier and Thomas G. Kurtz. The infinitely-many-neutral-alleles diffusion model. Adv. in Appl. Probab. 13 (3), 429-452 (1981). MR615945.

S. N. Ethier and Thomas G. Kurtz. Fleming-Viot processes in population genetics. SIAM J. Control Optim. 31 (2), 345-386 (1993). MR1205982.

W. J. Ewens. Mathematical population genetics. I, volume 27 of Interdisciplinary Applied Mathematics. Springer-Verlag, New York, second edition (2004). ISBN 0-387-20191-2. Theoretical introduction.

S. Feng and F.-Y. Wang. A class of infinite-dimensional diffusion processes with connection to population genetics. J. Appl. Probab. 44 (4), 938-949 (2007). MR2382936.

S. Feng and F.-Y. Wang. Harnack inequality and applications for infinitedimensional GEM processes. Potential Anal. 44 (1), 137-153 (2016). MR3455213.

W. H. Fleming and M. Viot. Some measure-valued Markov processes in population genetics theory. Indiana Univ. Math. J. 28 (5), 817-843 (1979). MR542340.

M. Jacobsen. Examples of multivariate diffusions: time-reversibility; a CoxIngersoll-Ross type process (2001). Department of Theoretical Statistics, Preprint 6, University of Copenhagen.

N. L. Johnson. An approximation to the multinomial distribution: some properties and applications. Biometrika 47, 93-102 (1960). MR0111099.

L. Miclo. About projections of logarithmic Sobolev inequalities. In Séminaire de Probabilités, XXXVI, volume 1801 of Lecture Notes in Math., pages 201-221. Springer, Berlin (2003a). MR1971587.

L. Miclo. Sur l'inégalité de Sobolev logarithmique des opérateurs de Laguerre à petit paramètre. In Séminaire de Probabilités, XXXVI, volume 1801 of Lecture Notes in Math., pages 222-229. Springer, Berlin (2003b). MR1971588.

J. E. Mosimann. On the compound multinomial distribution, the multivariate $\beta$ distribution, and correlations among proportions. Biometrika 49, 65-82 (1962). MR0143299.

W. Stannat. On the validity of the log-Sobolev inequality for symmetric FlemingViot operators. Ann. Probab. 28 (2), 667-684 (2000). MR1782270.

F.-Y. Wang. Functional inequalities for empty essential spectrum. J. Funct. Anal. 170 (1), 219-245 (2000a). MR1736202.

F.-Y. Wang. Functional inequalities, semigroup properties and spectrum estimates. Infin. Dimens. Anal. Quantum Probab. Relat. Top. 3 (2), 263-295 (2000b). MR1812701. 